Reprod. Nutr. Dévelop., 1988, 28 (1), 197-198.

\title{
Effets de la nature et de la quantité d'énergie ingérée sur les dépôts adipeux et leur activité LPL chez le chevreau non sevré
}

\author{
P. BAS, P. SCHMIDELY, P. MORAND-FEHR, J. HERVIEU
}

Station de nutrition et alimentation II.N.R.A. de I'INA-PG, 16. rue Claude Bernard, 75231 Paris Cedex 05.

Summary. For the same level of metabolisable energy (ME) intake, male kids fed with a lactose rich milk replacer (group L) had a better average daily gain than with a fat rich milk replacer (group MG). The perirenal weight (PR) was heavier and the lipid content of omental adipose tissue (OM) higher in kids of group MG. The lipid content of PR and OM but not of sternal adipose tissue (ST) increased in relation with ME. The LPL activities of OM, PR and ST did not differ between these two groups.

La qualité des carcasses de chevreau de race laitière étant abaissée par un faible état d'engraissement et en particulier par un manque de dépôts adipeux de couverture, nous avons étudié les répercussions de l'ingestion de laits de teneurs variables en matières grasses (MG) et en énergie, sur la quantité de lipides déposés dans différents tisseux adipeux, et sur l'activité de leur lipoprotéine lipase (LPL).

Matériel et méthodes. Quinze chevreaux mâles de race Alpine ou Saanen sont alimentés de l'âge de 7 jours jusqu'à l'abattage (7 semaines) exclusivement soit avec du lait de remplacement enrichi en lactose grâce à du sérum acide de fromagerie [lot $\mathrm{L}, \mathrm{n}=6,35,1 \mathrm{~g}$ lactose, $15,9 \mathrm{~g}$ de $M G, 23,4 \mathrm{~g}$ de protéines, $437 \mathrm{Kcal}$ d'énergie métabolisable calculée (EM) pour $100 \mathrm{~g} \mathrm{MSl}$, soit avec un lait réengraissé avec une forte proportion de suif (lot $M G, n=9,25,0 \mathrm{~g}$ lactose, $27,0 \mathrm{~g} \mathrm{MG}, 23,2 \mathrm{~g}$ protéines, $501,5 \mathrm{Kcal} \mathrm{EM} / 100 \mathrm{~g} \mathrm{MS})$. Les teneurs en lipides et les activités LPL (Chilliard, Dorléans et Fehr, 1977) sont déterminées sur des échantillons des tissus adipeux omental (OM), périrénal (PR) et sternal (ST).

Résultats. Les quantités moyennes d'EM ingérées par jour et les gains de poids moyens quotidiens (GMQ) des 2 lots sont comparables (tabl. 1). Ces deux paramètres sont corrélés positivement $[r=+0,940, n=15$, écart-type résiduel (ETR $=11,0 \mathrm{~g} / \mathrm{j}, P<0,01 \mathrm{l}$. Cependant, en utilisant l'EM comme covariable, les GMQ ajustés du lot $L$ apparaissent supérieurs à ceux du lot $M G(+5 \%$, ETR = $10,6 \mathrm{~g} / \mathrm{j}, \mathrm{P}<0,10)$.

Les chevreaux du lot MG présentent des dépôts $P R$ significativement $(P<0,05)$ plus développés que ceux du lot $L$ (tabl. 1). Les poids des tissus $O M$ et $P R$ sont significativement liés à l'EM ingérée $(r=+0,907, E T R=38,0 \mathrm{~g}$, $P<0,01$ et $r=+0,662$, ETR $=45,4 \mathrm{~g}, P<0,01$ respectivement $)$ mais non ceux du tissu ST. A même niveau d'EM ingérée, le poids de PR est plus faible $(-36 \%, E T R=39,0 \mathrm{~g}, P<0,05)$ dans le lot $L$ que dans le lot $M G$. Les teneurs en lipides des tissus OM, PR et ST sont plus élevées dans le lot MG mais significativement $(P<0,05)$ dans $O M$ seulement. Elles s'accroissent avec le niveau énergétique dans les tissus $O M$ et $P R(P<0,05)$ mais non dans $S T$. Dans le tissu OM, la différence entre les 2 lots persiste au bénéfice du lot MG lorsque 
ceux-ci sont comparés à même niveau énergétique $(+4,8 \%, P<0,05)$. Cependant la quantité de lipides du tissu OM, n'est pas significativement plus élevée dans le lot MG, contrairement au tissu PR (+ $55 \%, P<0,05)$.

TABL. 1. - Performances zootechniques des chevreaux et caractéristiques des tissus adipeux omental, périrénal et sternal.

\begin{tabular}{|c|c|c|c|c|c|c|}
\hline & & \multicolumn{2}{|c|}{$\begin{array}{c}\text { Régime : Lactose } \\
n=6\end{array}$} & \multicolumn{3}{|c|}{$\begin{array}{c}\text { Régime : Lipides } \\
n=9\end{array}$} \\
\hline \multicolumn{2}{|c|}{$\begin{array}{l}\text { Energie ingérée (Mcal EM/j) } \\
\text { Croît journalier } \mathrm{g} / \mathrm{j}\end{array}$} & $\begin{array}{c}1^{\bar{x}}, 33 \\
213^{1,33}\end{array}$ & $\begin{array}{cc} & s \\
\pm \quad & 0,187 \\
\pm & 38,4\end{array}$ & $\begin{array}{c}\bar{x} \\
21,38\end{array}$ & $\begin{array}{l} \pm \\
\pm\end{array}$ & $\begin{array}{c}\text { s } \\
0,164 \\
30,7\end{array}$ \\
\hline Tissu omental & $\begin{array}{l}\text { Poids g } \\
\text { Lipides \% } \\
\text { LPL }\end{array}$ & $\begin{array}{l}163 \\
81,0 \\
275\end{array}$ & $\begin{array}{lc} \pm & 96,1 \\
\pm & 4,39 \\
\pm & 126\end{array}$ & $\begin{array}{l}200 \\
85,8^{*} \\
336\end{array}$ & $\begin{array}{l} \pm \\
\pm \\
\pm\end{array}$ & $\begin{array}{c}82,4 \\
3,60 \\
190\end{array}$ \\
\hline Tissu périrénal & $\begin{array}{l}\text { Poids g } \\
\text { Lipides \% } \\
\text { LPL }\end{array}$ & $\begin{array}{l}89 \\
80,9 \\
279\end{array}$ & $\begin{array}{lc} \pm & 52,1 \\
\pm & 5,24 \\
\pm & 118\end{array}$ & $\begin{array}{c}148^{*} \\
85,2 \\
321\end{array}$ & $\begin{array}{l} \pm \\
\pm \\
\pm\end{array}$ & $\begin{array}{c}51,8 \\
6,53 \\
190\end{array}$ \\
\hline Tissu sternal & $\begin{array}{l}\text { Poids g } \\
\text { Lipides \% } \\
\text { LPL }\end{array}$ & $\begin{array}{c}12,9 \\
42,5 \\
176\end{array}$ & $\begin{array}{l} \pm \quad 6,49 \\
\pm \quad 8,32 \\
\pm \quad 57,1\end{array}$ & $\begin{array}{l}16,0 \\
44,5 \\
115\end{array}$ & $\begin{array}{l} \pm \\
\pm \\
\pm\end{array}$ & $\begin{array}{c}8,40 \\
7,74 \\
87,2\end{array}$ \\
\hline
\end{tabular}

Lipides : \%/tissu frais ; LPL : Lipoprotéine lipase $(\mathrm{nm} / \mathrm{mn} / \mathrm{g}$ de lipides $) ; \bar{x}:$ moyenne ; s : écart-type ; * : Différence entre les 2 lots significative au seuil de $5 \%$

Les activités LPL (par $\mathrm{g}$ de tissu ou de lipide) ne diffèrent pas selon les lots et ne semblent pas influencées ni par la quantité d'énergie ni par celle de lipides ingérés par jour. Cependant, l'activité LPL totale du tissu OM et dans une moindre mesure celle du tissu PR paraissent liées à la quantité de lipides ingérés avant l'abattage $\langle r=+0,707, P<0,01$ et $r=+0,603, P<0,01$ respectivement). Avec cette covariable, dans OM la LPL totale du lot 1 est supérieure à celle du lot $2(+57 \%, E T R=16,3 \mu \mathrm{eq} / \mathrm{mn}, P<0,05)$. Mais en prenant l'EM et la quantité de lipides ingérés avant l'abattage comme covariables, seule l'EM ingérée avant l'abattage a un effet significatif ( $P<0,01)$ sur l'activité LPL totale de OM. La liaison entre l'activité LPL totale de OM est moins étroite avec le poids de ce tissu qu'avec l'EM ingérée $l r=+0,568$, ETR = $23,1 \mu \mathrm{eq} / \mathrm{mn}, \mathrm{P}<0,05)$.

A ce stade de développement précoce, l'influence de la quantité d'EM ingérée sur le développement des tissus adipeux internes uniquement, confirme les résultats de Bas et al., (1987) sur les tissus adipeux intermusculaires et souscutanés du gigot. L'activité de la LPL du tissu adipeux est plus faible que celle observée chez des chevreaux non sevrés dont la vitesse de croissance était plus rapide (Bas, Rouzeau et Morand-Fehr, 1986) et qui ingéraient probablement plus d'énergie. La différence d'activité LPL entre les 2 lots est plus réduite que celle obtenue par Yang, Baldwin et Garrett (1978) sur bovins sevrés malgré leur différence importante d'apport de matières grasses. Avec ce type de régime riche en glucides, les LPL des 2 lots pourraient être stimulées de façon comparable par le glucose et l'insuline (Austin et Nestel, 1968).

Austin W., Nestel P. J., 1968. Biochim. Biophys. Acta, 164, 59-63.

Bas P., Rouzeau A., Morand-Fehr P., 1986. Reprod. Nutr. Dévelop., 26, 649-653.

Bas P., Morand-Fehr P., Schmidely P., Hervieu J., 1987. Ann. Zootech. (in press).

Chilliard Y., Dorléans M., Fehr P. M., 1977. Ann. Biol. anim. Bioch. Biophys., 17, 107-122.

Yang Y. T., Baldwin R. L., Garrett W. N., 1978. J. anim. Sci., 47, 686-690. 